VOL. 60 (1999) [469-477]

\title{
IDENTITIES IN ALGEBRAS WITH INVOLUTION
}

\author{
TSETSKa Grigorova RashKova
}

There is an approach due to Bergman describing ordinary polynomial identities for $M_{n}(K)$ by means of commutative algebra. In the paper we apply this approach to the matrix algebra of even order $M_{2 n}(K, *)$ with symplectic involution *.

In 1981 Bergman wrote a paper [1] which for some reasons remained unpublished. Nevertheless since then the preprint has been cited many times becoming a classic: both for the methods introduced and the main theorems proved, details of which will be stated later.

The present paper applies the ideas, introduced by Bergman for ordinary identities, to the study of $*$-identities in skew symmetric variables (* is the symplectic involution) and continues the investigations from [5].

The notion of *-polynomial identities for an associative algebra $R=(R, *)$ with involution $*$ is similar to that of ordinary polynomial identities [6].

Let $X \cup X^{*}=\left\{x_{1}, x_{2}, \ldots\right\} \cup\left\{x_{1}^{*}, x_{2}^{*}, \ldots\right\}$ and $K\left\langle X \cup X^{*}\right\rangle$ be the free associative algebra over a field $K$ of characteristic 0 with involution, defined by $(x)^{* *}=x,\left(x_{1}+x_{2}\right)^{*}=$ $x_{1}^{*}+x_{2}^{*},(\alpha x)^{*}=\alpha x^{*},\left(x_{1} x_{2}\right)^{*}=x_{2}^{*} x_{1}^{*}, x, x_{1}, x_{2} \in X \cup X^{*}, \alpha \in K$.

If we put

$$
y_{j}=x_{j}+x_{j}^{*}, z_{j}=x_{j}-x_{j}^{*}, j=1,2, \ldots,
$$

then $K\left\langle X \cup X^{*}\right\rangle=K\langle Y \cup Z\rangle$, where $Y=\left\{y_{1}, y_{2}, \ldots\right\}$ and $Z=\left\{z_{1}, z_{2}, \ldots\right\}$.

Let $R$ be an associative algebra with involution. As $r=1 / 2\left(r+r^{*}\right)+1 / 2\left(r-r^{*}\right)$, for all $r$ in $R$, the algebra $(R, *)$ is a direct sum of subspaces, that is, $(R, *)=R^{+} \oplus R^{-}$, where $R^{ \pm}=\left\{r \in R \mid r^{*}= \pm r\right\}$.

An element $f\left(y_{1}, \ldots, y_{m}, z_{1}, \ldots, z_{n}\right)$ of $K\langle Y \cup Z\rangle$ is a *-polynomial identity for $(R, *)$ if $f\left(r_{1}^{+}, \ldots, r_{m}^{+}, r_{1}^{-}, \ldots, r_{n}^{-}\right)=0$ for any $r_{i}^{+} \in R^{+}, r_{j}^{-} \in R^{-}, i=1, \ldots, m ; j=1, \ldots, n$.

We are interested in *-identitities in skew symmetric variables of the following type, considered in the ordinary case by Formanek [3] and Bergman [1] and by the author and Drensky [2] in the case of weak polynomial identities for Lie algebras.

Received 25th February, 1999

Partially supported by Grant MM605/96 of the Bulgarian Foundation for Scientific Research. I thank $V$. Drensky for directing my attention to the problem and the useful suggestions made and to G. Ivanov for helping me to improve the style of the paper.

Copyright Clearance Centre, Inc. Serial-fee code: 0004-9727/99 \$A2.00+0.00. 
To a polynomial in commuting variables

$$
g\left(t_{1}, \ldots, t_{n+1}\right)=\sum \alpha_{p} t_{1}^{p_{1}} \ldots t_{n+1}^{p_{n+1}} \in K\left[t_{1}, \ldots, t_{n+1}\right]
$$

we relate a polynomial $v(g)$ from the free associative algebra $K\left\langle x, y_{1}, \ldots, y_{n}\right\rangle$

$$
\begin{aligned}
v(g) & =v(g)\left(x, y_{1}, \ldots, y_{n}\right) \\
& =\sum \alpha_{p} x^{p_{1}} y_{1} x^{p_{2}} y_{2} \ldots x^{p_{n}} y_{n} x^{p_{n+1}}
\end{aligned}
$$

Any polynomial $f\left(x, y_{1}, \ldots, y_{n}\right)$ multilinear in $y_{1}, \ldots, y_{n}$ can be written as

$$
\begin{aligned}
f\left(x, y_{1}, \ldots, y_{n}\right)=\sum \beta_{i} v\left(g_{i}\right)\left(x, y_{i_{1}}, \ldots, y_{i_{n}}\right), & \\
& \beta_{i} \in K, g_{i} \in K\left[t_{1}, \ldots, t_{n+1}\right] .
\end{aligned}
$$

There is a criterion due to Bergman [1] describing the identities for $M_{n}(K)$ of type (3) by the properties of the polynomials in commuting variables of type (1).

BERGMAN'S CRITERION (BC)

(i) $[1$, Section $6,(27)]$ The polynomial $v(g)\left(x, y_{1}, \ldots, y_{n}\right)$ from (2) is an identity for $M_{n}(K)$ if and only if

$$
\prod_{1 \leqslant p<q \leqslant n+1}\left(t_{p}-t_{q}\right)
$$

divides the polynomial $g\left(t_{1}, \ldots, t_{n+1}\right)$ from (1).

(ii) $[1$, Section $7,(38)]$ The polynomial $f\left(x, y_{1}, \ldots, y_{n}\right)$ from (3) is an identity for $M_{n}(K)$ if and only if every summand $v\left(g_{i}\right)$ is also an identity for $M_{n}(K)$.

Since [1] is in a preprint form, we refer to [2, Proposition 1.2] for the proof of $\mathbf{B C}$.

Our aim is to find the statement corresponding to $\mathbf{B C}$ for *-polynomial identities in skew symmetric variables for the $2 n \times 2 n$ matrix algebra $M_{2 n}(K, *)$ with symplectic involution $*$ defined by

$$
\left(\begin{array}{cc}
A & B \\
C & D
\end{array}\right)^{*}=\left(\begin{array}{cc}
D^{t} & -B^{t} \\
-C^{t} & A^{t}
\end{array}\right),
$$

where $A, B, C, D$ are $n \times n$ matrices and $t$ is the usual transpose. For background on *-polynomial identities for $M_{2 n}(K, *)$ see for example, [4].

In [5] the author and $\mathrm{V}$. Drensky proved an extension of $\mathrm{BC}$ giving a necessary and sufficient condition the polynomials $v(g)\left(x, y_{1}, \ldots, y_{n}\right)$ to be *-identities in skew symmetric variables for $M_{2 n}(K, *)$. However we found an example for $n=2$ showing that in the case considered a polynomial of type (3) is a $*$-identity while its parts $v\left(g_{i}\right)$ are not $*$-identities themselves.

Let us consider the polynomial $f=\left[\left[x^{2}, y\right]^{2}, x\right]$. According to [4] $f=0$ is a *-identity in skew symmetric variables for $M_{4}(K, *)$. Its linearisation in $y$, which is such an identity 
too, could be written as

$$
\begin{aligned}
\operatorname{lin}_{y} f & =f_{1}+f_{2}, \text { where } \\
f_{1} & =\sum_{p=\left(p_{1}, p_{2}, p_{3}\right) \vdash 4} \alpha_{p} x^{p_{1}} y_{1} x^{p_{2}} y_{2} x^{p_{3}} \text { and } \\
f_{2} & =\sum_{p=\left(p_{1}, p_{2}, p_{3}\right) \vdash 4} \alpha_{p} x^{p_{1}} y_{2} x^{p_{2}} y_{1} x^{p_{3}}, \alpha_{p} \in K .
\end{aligned}
$$

We calculate $f_{i}\left(\rho_{1}\left(e_{11}-e_{33}\right)+\rho_{2}\left(e_{22}-e_{44}\right), e_{12}-e_{43}, e_{14}+e_{23}\right)$ for $i=1,2$ and get

$$
\begin{aligned}
& f_{1}=2 \rho_{1}\left(\rho_{1}^{2}-\rho_{2}^{2}\right)^{2} e_{13} \neq 0 \text { and } \\
& f_{2}=-2 \rho_{1}\left(\rho_{1}^{2}-\rho_{2}^{2}\right)^{2} e_{13} \neq 0 .
\end{aligned}
$$

Thus $f_{i}(i=1,2)$ are not $*$-identities in skew symmetric variables (as the coefficients $\rho_{1}, \rho_{2}$ are arbitrary).

The next theorem is the main result of the paper.

THEOREM 1. Let the polynomial $f\left(x, y_{1}, \ldots, y_{n}\right)$ of type (3) be a $*$-identity in skew symmetric variables for $M_{2 n}(K, *)$. Then

$$
\prod_{\substack{1 \leq p<q \leqslant n+1 \\(p, q) \neq(1, n+1)}}\left(t_{p}^{2}-t_{q}^{2}\right)\left(t_{1}-t_{n+1}\right)
$$

divides the polynomials $g_{i}$ from (1) for all $i=\left(i_{1}, \ldots, i_{n}\right)$.

Our investigation is based on $\mathbf{B C}$ and the next two propositions, the first of which is well known.

Proposition 1. Every *-identity in skew symmetric variables for $M_{2 n}(K, *)$ is an ordinary polynomial identity for $M_{n}(K)$.

Proposition 2. [5, Theorem 1] The polynomial $v(g)\left(x, y_{1}, \ldots, y_{n}\right)$ from (2) is a *-polynomial identity in skew symmetric variables for $M_{2 n}(K, *)$ if and only if $\prod_{1 \leqslant p<q \leqslant n+1}\left(t_{p}^{2}-t_{q}^{2}\right)$ divides the polynomial $g\left(t_{1}, \ldots, t_{n+1}\right)$ from (1).

In Proposition 2 the special ordering of the $y^{\prime}$ s in the polynomial from (2) is not essential.

LEMMA 1. The polynomials $v\left(g_{i}\right)\left(x, y_{i_{1}}, \ldots, y_{i_{n}}\right)$ from (3) are *-polynomial identities in skew symmetric variables for $M_{2 n}(K, *)$ if and only if $\prod_{1 \leqslant p<q \leqslant n+1}\left(t_{p}^{2}-t_{q}^{2}\right)$ divides the polynomials $g_{i}\left(t_{1}, \ldots, t_{n+1}\right)$ from (1) for all $i=\left(i_{1}, \ldots, i_{n}\right)$.

Proof: It follows the proof of Proposition 2 closely, changing only the indices of $y_{i}$ (we replace $y_{1}, \ldots, y_{n}$ with $y_{i_{1}}, \ldots, y_{i_{n}}$, respectively). 
Proof of Theorem 1: Because of Proposition 2 and Lemma 1 we have to prove that if any polynomial $f\left(x, y_{1}, \ldots, y_{n}\right)$ is a $*$-identity then $\prod_{\substack{1 \leqslant p<q \leqslant n+1 \\ 1}}\left(t_{p}+t_{q}\right)$ divides the polynomials $g_{i}\left(t_{1}, \ldots, t_{n+1}\right)$ from (1) for all $i=\left(i_{1}, \ldots, i_{n}\right)$.

Let the polynomial $f\left(x, y_{1}, \ldots, y_{n}\right)$ be a $*$-identity.

1 (a) We make the following substitution:

(4)

$$
\begin{aligned}
\bar{x}= & \sum_{i=1}^{n} \rho_{i}\left(e_{i i}-e_{n+i, n+i}\right), \\
\bar{y}_{1}= & e_{12}-e_{n+2, n+1}, \\
& \vdots \\
\bar{y}_{n-1}= & e_{n-1, n}-e_{2 n, 2 n-1} \\
\bar{y}_{n}= & e_{n, 2 n} .
\end{aligned}
$$

Due to the staircase lemma [6, p.22] the only non-zero summands of $f$ will be

$$
\sum \alpha_{p} x^{p_{1}} y_{1} x^{p_{2}} y_{2} \ldots y_{n} x^{p_{n+1}}, \alpha_{p} \in K
$$

and

$$
\sum \beta_{p} x^{p_{1}} y_{n} x^{p_{2}} y_{n-1} \ldots y_{1} x^{p_{n+1}}, \beta_{p} \in K
$$

Then

$$
\begin{aligned}
0=f\left(\bar{x}, \bar{y}_{i_{1}}, \ldots, \bar{y}_{i_{n}}\right)=g_{\alpha} & \left(\rho_{1}, \rho_{2}, \ldots, \rho_{n},-\rho_{n}\right) e_{1,2 n} \\
& +(-1)^{n-1} g_{\beta}\left(\rho_{n},-\rho_{n},-\rho_{n-1}, \ldots,-\rho_{1}\right) e_{n, n+1} .
\end{aligned}
$$

Hence

$$
\begin{aligned}
g_{\alpha}\left(\rho_{1}, \rho_{2}, \ldots, \rho_{n},-\rho_{n}\right)=0 & \text { and } \\
g_{\beta}\left(\rho_{n},-\rho_{n},-\rho_{n-1}, \ldots,-\rho_{1}\right)=0 . &
\end{aligned}
$$

This means that $t_{n}+t_{n+1}$ divides $g_{\alpha}$ and $t_{1}+t_{2}$ divides $g_{\beta}$.

If the substitution from (4) is for $\bar{x}, \bar{y}_{n}, \ldots, \bar{y}_{1}$, respectively, we get that $t_{n}+t_{n+1}$ divides $g_{\beta}$ and $t_{1}+t_{2}$ divides $g_{\alpha}$.

1(b) Obviously when only (5) is a summand of $f$ to have $t_{1}+t_{2}$ dividing $g_{\beta}$ we have to make in (5) the substitution

$$
\begin{aligned}
\bar{x}= & \sum_{i=1}^{n} \rho_{i}\left(e_{i i}-e_{n+i, n+i}\right), \\
\bar{y}_{1}= & e_{n, 2 n}, \\
\bar{y}_{2}= & e_{n-1, n}-e_{2 n, 2 n-1}, \\
& \vdots \\
\bar{y}_{n}= & e_{12}-e_{n+2, n+1} .
\end{aligned}
$$


We work analogously when only (6) is a part of $f$. When neither of these two summands is a part of $f$ we consider the pair $\sum \gamma_{p} x^{p_{1}} y_{i_{1}} x^{p_{2}} y_{i_{2}} \ldots y_{i_{n}} x^{p_{n+1}}$ and $\sum \delta_{p} x^{p_{1}} y_{i_{n}} x^{p_{2}} y_{i_{n-1}} \ldots y_{i_{1}} x^{p_{n+1}}$ and in this case the corresponding substitutions are for $\bar{y}_{i j}, j=1, \ldots, n$.

2(a) If we make $n-2$ more substitutions, changing only $\bar{y}_{n}$ in (4), namely $\bar{y}_{n}=$ $e_{n, n+k}+e_{k, 2 n}$ for $k=2, \ldots, n-1$, we come to

$$
\begin{aligned}
0=f\left(\bar{x}, \bar{y}_{1}, \ldots, \bar{y}_{n}\right)=g_{\alpha}\left(\rho_{1}, \rho_{2}, \ldots, \rho_{n},-\rho_{k}\right) e_{1, n+k} \\
\\
+(-1)^{n} g_{\beta}\left(\rho_{k},-\rho_{n},-\rho_{n-1}, \ldots,-\rho_{1}\right) e_{k, n+1} .
\end{aligned}
$$

Hence

$$
\begin{aligned}
g_{\alpha}\left(\rho_{1}, \rho_{2}, \ldots, \rho_{n},-\rho_{k}\right) & =0 \quad \text { and } \\
g_{\beta}\left(\rho_{k},-\rho_{n},-\rho_{n-1}, \ldots,-\rho_{1}\right) & =0 .
\end{aligned}
$$

From the first equality it follows that $t_{k}+t_{n+1}$ divides $g_{\alpha}$ for $k=2, \ldots, n-1$. If we rearrange the indices in the second equality we get $t_{1}+t_{n-k+2}$ dividing $g_{\beta}$.

2(b) When only one of the summands (5) or (6) is a part of $f$ the considerations are analogous to those in $1(\mathrm{~b})$.

3. Now we fix $i$ and $k(1 \leqslant i<k \leqslant n)$ and substitute

$$
\begin{aligned}
\bar{x}= & \rho_{1} e_{11}+\ldots+\rho_{k-1} e_{k-1, k-1}+\rho_{k+1} e_{k k}+\ldots+\rho_{n+1} e_{n n} \\
& -\rho_{1} e_{n+1, n+1}-\ldots-\rho_{k-1} e_{n+k-1, n+k-1} \\
& -\rho_{k+1} e_{n+k, n+k}-\ldots-\rho_{n+1} e_{2 n, 2 n} \\
\bar{y}_{1}= & e_{12}-e_{n+2, n+1} \\
\bar{y}_{2}= & e_{23}-e_{n+3, n+2} \\
& \vdots \\
\bar{y}_{k-2}= & e_{k-2, k-1}-e_{n+k-1, n+k-2} \\
\bar{y}_{k-1}= & e_{k-1, n+i}+e_{i, n+k-1} \\
\bar{y}_{k}= & e_{n+i, k}+e_{n+k, i} \\
\bar{y}_{k+1}= & e_{k, k+1}-e_{n+k+1, n+k} \\
& \vdots \\
\bar{y}_{n-1}= & e_{n-2, n-1}-e_{2 n-1,2 n-2} \\
\bar{y}_{n}= & e_{n-1, n}-e_{2 n, 2 n-1} .
\end{aligned}
$$

Thus we have

$$
\begin{aligned}
0= & f\left(\bar{x}, \bar{y}_{1}, \ldots, \bar{y}_{n}\right) \\
= & g_{\alpha}\left(\rho_{1}, \ldots, \rho_{k-1},-\rho_{i}, \rho_{k+1}, \ldots, \rho_{n+1}\right) e_{1, n} \\
& \quad+(-1)^{n-2} g_{\beta}\left(-\rho_{n+1},-\rho_{n}, \ldots,-\rho_{k+1}, \rho_{i},-\rho_{k-1}, \ldots,-\rho_{1}\right) e_{2 n, n+1} .
\end{aligned}
$$


This means that

$$
\begin{aligned}
g_{\alpha}\left(\rho_{1}, \ldots, \rho_{k-1},-\rho_{i}, \rho_{k+1}, \ldots, \rho_{n+1}\right)=0 & \text { and } \\
g_{\beta}\left(-\rho_{n+1},-\rho_{n}, \ldots,-\rho_{k+1}, \rho_{i},-\rho_{k-1}, \ldots,-\rho_{1}\right)=0 &
\end{aligned}
$$

From the first equality we get that $t_{i}+t_{k}$ divides $g_{\alpha}$ for $i=1,2, \ldots, k-1$. Rearranging the indices in the second equality we see that $t_{i}+t_{n-k+2}$ divides $g_{\beta}$.

Following 1(a) all the properties obtained in the sequel are both for $g_{\alpha}$ and $g_{\beta}$.

Allowing $k$ to be successively $2, \ldots, n$ we get finally that $\prod_{p}\left(t_{p}+t_{q}\right)$ divides $g_{\alpha}$ and $g_{\beta}$.

$$
\begin{aligned}
& 1 \leqslant p<q \leqslant n+1 \\
& (p, q) \neq(1, n+1)
\end{aligned}
$$

Thus $\prod_{\substack{1 \leqslant p<q \leqslant n+1 \\(p, q) \neq(1, n+1)}}\left(t_{p}^{2}-t_{q}^{2}\right)\left(t_{1}-t_{n+1}\right)$ divides the polynomials $g_{\alpha}$ and $g_{\beta}$.

In the same way we get the validity of the theorem for $g_{i}\left(t_{1}, \ldots, t_{n+1}\right)$ for all $i=$ $\left(i_{1}, \ldots, i_{n}\right)$.

REMARK 1. If in addition $t_{1}+t_{n+1}$ divides $g_{i}$ then $v\left(g_{i}\right)$ are $*$-identities for all $i=$ $\left(i_{1}, \ldots, i_{n}\right)$.

REMARK 2. Theorem 1 shows that the minimal possible degree of a $*$-identity of the considered type is $n^{2}+2 n-1$. It is exactly $n^{2}+2 n-1$ for $n=3$.

Let

$$
g=\prod_{\substack{1 \leq p<q \leqslant 4 \\(p, q) \neq(1,4)}}\left(t_{p}^{2}-t_{q}^{2}\right)\left(t_{1}-t_{4}\right)
$$

and

$$
B\left(x, y_{1}, y_{2}, y_{3}\right)=\sum_{\sigma \in S y m(3)} v(g)\left(x, y_{\sigma(1)}, y_{\sigma(2)}, y_{\sigma(3)}\right)
$$

be the associative polynomial corresponding to it from (3).

THEOREM 2. The polynomial $B\left(x, y_{1}, y_{2}, y_{3}\right)$ is a *-identity in skew symmetric variables for $M_{6}(K, *)$.

We need an intermediate result.

\section{THEOREM 3. The polynomial}

$$
B_{1}\left(x, y_{1}, y_{2}, y_{3}\right)=v(g)\left(x, y_{1}, y_{2}, y_{3}\right)+v(g)\left(x, y_{3}, y_{2}, y_{1}\right)
$$

is a *-identity in skew symmetric variables for $M_{6}(K, *)$.

Proof: The polynomial $B_{1}\left(x, y_{1}, y_{2}, y_{3}\right)$ is multilinear in $y^{\prime}$ s. Thus in order to see that it is a $*$-identity, according to $[5$, Proposition 4$]$ it is enough to take $\bar{x}=\sum_{i=1}^{3} \rho_{i}\left(e_{i i}-\right.$ $\left.e_{3+i, 3+i}\right)$ and $\bar{y}_{s}^{\prime}$ to be skew symmetric matrix units.

Due to the staircase lemma the cases are classified as follows: 
I. Let $y_{1}$ be a diagonal matrix.

1. $y_{1}=e_{i i}-e_{3+i, 3+i}, y_{2}=e_{i j}-e_{3+j, 3+i}(i \neq j)$, $y_{3} \in\left\{e_{k, 3+j}+e_{j, 3+k}, e_{j k}-e_{3+k, 3+j}(j \neq k), e_{j j}-e_{3+j, 3+j}, e_{j, 3+j}\right\}$;

2. $y_{1}=e_{i i}-e_{3+i, 3+i}, y_{2}=e_{i, 3+j}+e_{j, 3+i}(i \neq j)$, $y_{3} \in\left\{e_{3+j, k}+e_{3+k, j}, e_{k j}-e_{3+j, 3+k}(j \neq k), e_{j j}-e_{3+j, 3+j}, e_{3+j, j}\right\}$;

3. $y_{1}=e_{i i}-e_{3+i, 3+i}, y_{2}=e_{3+i, j}+e_{3+j, i}(i \neq j)$, $y_{3} \in\left\{e_{j, 3+k}+e_{k, 3+j}, e_{j k}-e_{3+k, 3+j}(j \neq k), e_{j j}-e_{3+j, 3+j}, e_{j, 3+j}\right\}$;

4. $y_{1}=e_{i i}-e_{3+i, 3+i}, y_{2}=e_{i, 3+i}, y_{3} \in\left\{e_{k i}-e_{3+i, 3+k}, e_{3+i, k}+e_{3+k, i}(i \neq k), e_{3+i, i},\right\}$;

5. $y_{1}=e_{i i}-e_{3+i, 3+i}, y_{2}=e_{3+i, i}, y_{3} \in\left\{e_{i k}-e_{3+k, 3+i}, e_{i, 3+k}+e_{k, 3+i}(i \neq k), e_{i, i+3},\right\}$.

II. If $y_{1}$ is not a diagonal matrix we have:

6. $y_{1}=e_{i j}-e_{3+j, 3+i}(i \neq j), y_{2}=e_{j j}-e_{3+j, 3+j}$, $y_{3} \in\left\{e_{k, 3+j}+e_{j, 3+k}, e_{j k}-e_{3+k, 3+j}(j \neq k), e_{j, 3+j}\right\}$

7. $y_{1}=e_{i j}-e_{3+j, 3+i}(i \neq j), y_{2}=e_{k, 3+j}+e_{j, 3+k}(j \neq k)$, $y_{3} \in\left\{e_{3+k, l}+e_{3+l, k}, e_{l k}-e_{3+k, 3+l}, e_{k k}-e_{3+k, 3+k}(l \neq k), e_{3+k, k}\right\}$

8. $y_{1}=e_{i j}-e_{3+j, 3+i}(i \neq j), y_{2}=e_{j k}-e_{3+k, 3+j}(j \neq k)$, $y_{3} \in\left\{e_{k, 3+l}+e_{l, 3+k}, e_{l k}-e_{3+k, 3+l}, e_{l l}-e_{3+l, 3+l}(l \neq k), e_{l, 3+l}\right\}$;

9. $y_{1}=e_{i j}-e_{3+j, 3+i}(i \neq j), y_{2}=e_{j, 3+k}-e_{k, 3+j}(j \neq k)$, $y_{3} \in\left\{e_{3+k, l}+e_{3+l, k}, e_{l k}-e_{3+k, 3+l}(l \neq k), e_{k k}-e_{3+k, 3+k}, e_{3+k, k}\right\}$;

10. $y_{1}=e_{i j}-e_{3+j, 3+i}(i \neq j), y_{2}=e_{j, 3+j}$, $y_{3} \in\left\{e_{3+j, k}+e_{3+k, j}, e_{k j}-e_{3+j, 3+k}(j \neq k), e_{j j}-e_{3+j, 3+j}, e_{3+j, j}\right\} ;$

11. $y_{1}=e_{i j}-e_{3+j, 3+i}, y_{2}=e_{3+i, j}+e_{3+j, i}(i \neq j)$,

$y_{3} \in\left\{e_{j, 3+k}+e_{k, 3+j}, e_{j k}-e_{3+k, 3+j}(j \neq k), e_{j j}-e_{3+j, 3+j}, e_{j, 3+j}\right\}$

12. $y_{1}=e_{i j}-e_{3+j, 3+i}(i \neq j), y_{2}=e_{i+3, i}$, $y_{3} \in\left\{e_{k, 3+i}+e_{i, 3+k}, e_{i k}-e_{3+k, 3+i}(i \neq k), e_{i i}-e_{3+i, 3+i}, e_{i, 3+i}\right\}$.

The considerations are similar in all cases and we shall show only two typical substitutions:

I. $y_{1}=e_{11}-e_{44}, y_{2}=e_{16}+e_{34}, y_{3}=e_{43}+e_{61}$. In this case

$$
\begin{aligned}
& x^{\alpha_{1}} y_{1}=\rho_{1}^{\alpha_{1}} e_{11}+\left(-\rho_{1}\right)^{\alpha_{1}} e_{44}, \\
& x^{\alpha_{1}} y_{1} x^{\alpha_{2}}=\rho_{1}^{\alpha_{1}+\alpha_{2}} e_{11}+\left(-\rho_{1}\right)^{\alpha_{1}+\alpha_{2}} e_{44}, \\
& x^{\alpha_{1}} y_{1} x^{\alpha_{2}} y_{2}=\rho_{1}^{\alpha_{1}+\alpha_{2}} e_{16}, \\
& x^{\alpha_{1}} y_{1} x^{\alpha_{2}} y_{2} x^{\alpha_{3}}=\rho_{1}^{\alpha_{1}+\alpha_{2}}\left(-\rho_{3}\right)^{\alpha_{3}} e_{16}, \\
& x^{\alpha_{1}} y_{1} x^{\alpha_{2}} y_{2} x^{\alpha_{3}} y_{3}=\rho_{1}^{\alpha_{1}+\alpha_{2}}\left(-\rho_{3}\right)^{\alpha_{3}} e_{11}, \\
& x^{\alpha_{1}} y_{1} x^{\alpha_{2}} y_{2} x^{\alpha_{3}} y_{3} x^{\alpha_{4}}=\rho_{1}^{\alpha_{1}+\alpha_{2}+\alpha_{4}}\left(-\rho_{3}\right)^{\alpha_{3}} e_{11} ; \\
& x^{\alpha_{1}} y_{3}=\left(-\rho_{1}\right)^{\alpha_{1}} e_{43}+\left(-\rho_{3}\right)^{\alpha_{1}} e_{61},
\end{aligned}
$$




$$
\begin{aligned}
& x^{\alpha_{1}} y_{3} x^{\alpha_{2}}=\left(-\rho_{1}\right)^{\alpha_{1}} \rho_{3}^{\alpha_{2}} e_{43}+\left(-\rho_{3}\right)^{\alpha_{1}} \rho_{1}^{\alpha_{2}} e_{61}, \\
& x^{\alpha_{1}} y_{3} x^{\alpha_{2}} y_{2}=\left(-\rho_{1}\right)^{\alpha_{1}} \rho_{3}^{\alpha_{2}} e_{44}, \\
& x^{\alpha_{1}} y_{3} x^{\alpha_{2}} y_{2} x^{\alpha_{3}}=\left(-\rho_{1}\right)^{\alpha_{1}} \rho_{3}^{\alpha_{2}}\left(-\rho_{1}\right)^{\alpha_{3}} e_{44}, \\
& x^{\alpha_{1}} y_{3} x^{\alpha_{2}} y_{2} x^{\alpha_{3}} y_{1}=-\left(-\rho_{1}\right)^{\alpha_{1}} \rho_{3}^{\alpha_{2}}\left(-\rho_{1}\right)^{\alpha_{3}} e_{44}, \\
& x^{\alpha_{1}} y_{3} x^{\alpha_{2}} y_{2} x^{\alpha_{3}} y_{1} x^{\alpha_{4}}=-\left(-\rho_{1}\right)^{\alpha_{1}} \rho_{3}^{\alpha_{2}}\left(-\rho_{1}\right)^{\alpha_{3}}\left(-\rho_{1}\right)^{\alpha_{4}} e_{44}
\end{aligned}
$$

Thus we get

$$
B_{1}\left(x, y_{1}, y_{2}, y_{3}\right)=g\left(\rho_{1}, \rho_{2},-\rho_{3}, \rho_{1}\right) e_{11}-g\left(-\rho_{1}, \rho_{3},-\rho_{1}, \rho_{1}\right) e_{44}
$$

It follows from the definition of $g\left(t_{1}, t_{2}, t_{3}, t_{4}\right)$ that

$$
\begin{array}{r}
g\left(\rho_{1}, \rho_{2},-\rho_{3}, \rho_{1}\right)=g\left(\rho_{1}, \rho_{2}, \rho_{3}, \rho_{1}\right)=0 \\
g\left(-\rho_{1}, \rho_{3},-\rho_{1},-\rho_{1}\right)=-g\left(\rho_{1}, \rho_{3}, \rho_{1}, \rho_{1}\right)=0
\end{array}
$$

and $B_{1}\left(x, y_{1}, y_{2}, y_{3}\right)=0$.

II. $y_{1}=e_{15}+e_{24}, y_{2}=e_{32}-e_{56}, y_{3}=e_{13}-e_{64}$. In this case

$$
\begin{aligned}
& x^{\alpha_{1}} y_{1}=\rho_{1}^{\alpha_{1}} e_{15}+\left(\rho_{2}\right)^{\alpha_{1}} e_{24} \\
& x^{\alpha_{1}} y_{1} x^{\alpha_{2}}=\rho_{1}^{\alpha_{1}}\left(-\rho_{2}\right)^{\alpha_{2}} e_{15}+\left(\rho_{2}\right)^{\alpha_{1}}\left(-\rho_{1}\right)^{\alpha_{2}} e_{24}, \\
& x^{\alpha_{1}} y_{1} x^{\alpha_{2}} y_{2}=-\rho_{1}^{\alpha_{1}}\left(-\rho_{2}\right)^{\alpha_{2}} e_{16} \\
& x^{\alpha_{1}} y_{1} x^{\alpha_{2}} y_{2} x^{\alpha_{3}}=-\rho_{1}^{\alpha_{1}}\left(-\rho_{2}\right)^{\alpha_{2}}\left(-\rho_{3}\right)^{\alpha_{3}} e_{16}, \\
& x^{\alpha_{1}} y_{1} x^{\alpha_{2}} y_{2} x^{\alpha_{3}} y_{3}=\rho_{1}^{\alpha_{1}}\left(-\rho_{2}\right)^{\alpha_{2}}\left(-\rho_{3}\right)^{\alpha_{3}} e_{14}, \\
& x^{\alpha_{1}} y_{1} x^{\alpha_{2}} y_{2} x^{\alpha_{3}} y_{3} x^{\alpha_{4}}=\rho_{1}^{\alpha_{1}}\left(-\rho_{2}\right)^{\alpha_{2}}\left(-\rho_{3}\right)^{\alpha_{3}}\left(-\rho_{1}\right)^{\alpha_{4}} e_{14} \\
& x^{\alpha_{1}} y_{3}=\rho_{1}^{\alpha_{1}} e_{13}-\left(-\rho_{3}\right)^{\alpha_{1}} e_{64}, \\
& x^{\alpha_{1}} y_{3} x^{\alpha_{2}}=\rho_{1}^{\alpha_{1}} \rho_{3}^{\alpha_{2}} e_{13}-\left(-\rho_{3}\right)^{\alpha_{1}}\left(-\rho_{1}\right)^{\alpha_{2}} e_{64}, \\
& x^{\alpha_{1}} y_{3} x^{\alpha_{2}} y_{2}=\rho_{1}^{\alpha_{1}} \rho_{3}^{\alpha_{2}} e_{12}, \\
& x^{\alpha_{1}} y_{3} x^{\alpha_{2}} y_{2} x^{\alpha_{3}}=\rho_{1}^{\alpha_{1}} \rho_{3}^{\alpha_{2}} \rho_{2}^{\alpha_{3}} e_{12}, \\
& x^{\alpha_{1}} y_{3} x^{\alpha_{2}} y_{2} x^{\alpha_{3}} y_{1}=\rho_{1}^{\alpha_{1}} \rho_{3}^{\alpha_{2}} \rho_{2}^{\alpha_{3}} e_{14}, \\
& x^{\alpha_{1}} y_{3} x^{\alpha_{2}} y_{2} x^{\alpha_{3}} y_{1} x^{\alpha_{4}}=\rho_{1}^{\alpha_{1}} \rho_{3}^{\alpha_{2}} \rho_{2}^{\alpha_{3}}\left(-\rho_{1}\right)^{\alpha_{4}} e_{14} .
\end{aligned}
$$

Thus

$$
B_{1}\left(x, y_{1}, y_{2}, y_{3}\right)=\left(g\left(\rho_{1},-\rho_{2},-\rho_{3},-\rho_{1}\right)+g\left(\rho_{1}, \rho_{3}, \rho_{2},-\rho_{1}\right)\right) e_{14}
$$

From the properties of $g\left(t_{1}, t_{2}, t_{3}, t_{4}\right)$ we get that

$$
B_{1}\left(x, y_{1}, y_{2}, y_{3}\right)=\left(g\left(\rho_{1}, \rho_{2}, \rho_{3},-\rho_{1}\right)-g\left(\rho_{1}, \rho_{2}, \rho_{3},-\rho_{1}\right)\right) e_{14}=0 .
$$

ProOF OF THEOREM 3: This follows immediately from the equality

$$
B\left(x, y_{1}, y_{2}, y_{3}\right)=B_{1}\left(x, y_{1}, y_{2}, y_{3}\right)+B_{1}\left(x, y_{2}, y_{1}, y_{3}\right)+B_{1}\left(x, y_{1}, y_{3}, y_{2}\right)
$$


COROLlary 1. The polynomial $B_{1}\left(x, y_{1}, y_{1}, y_{2}\right)$ is a *-identity in skew symmetric variables for $M_{6}(K, *)$.

Corollary 2. The polynomial $v(g)\left(x, y_{1}, y_{2}, y_{1}\right)$ is a *-identity in skew symmetric variables for $M_{6}(K, *)$.

COROLlary 3. The polynomial $v(g)\left(x, y_{1}, y_{1}, y_{1}\right)$ is a *-identity in skew symmetric variables for $M_{6}(K, *)$.

REMARK 3. The summands $v(g)\left(x, y_{i_{1}}, y_{i_{2}}, y_{i_{3}}\right)$ themselves are not *-identities for $M_{6}(K, *)$.

We check that $v(g)\left(\bar{x}, \bar{y}_{i_{1}}, \bar{y}_{i_{2}}, \bar{y}_{i_{3}}\right) \neq 0$ for

$$
\begin{gathered}
\bar{x}=e_{11}-e_{44}-2\left(e_{22}-e_{55}\right)+5\left(e_{33}-e_{66}\right), \\
\bar{y}_{i_{1}}=e_{12}-e_{54}, \bar{y}_{i_{2}}=e_{23}-e_{65}, \bar{y}_{i_{3}}=e_{16}+e_{34} .
\end{gathered}
$$

In this case $v(g)\left(\bar{x}, \bar{y}_{i_{1}}, \bar{y}_{i_{2}}, \bar{y}_{i_{3}}\right)=-217728 e_{14}$.

If we form

$$
B_{2}\left(x, y_{1}, y_{2}, y_{3}\right)=\sum_{\sigma \in S y m(3)}(-1)^{\sigma} v(g)\left(x, y_{\sigma(1)}, y_{\sigma(2)}, y_{\sigma(3)}\right)
$$

we see that this polynomial is not a *-identity as well. For example

$$
B_{2}\left(e_{11}-e_{44}-2\left(e_{22}-e_{55}\right)+5\left(e_{33}-e_{66}\right), e_{12}-e_{54}, e_{23}-e_{65}, e_{16}+e_{34}\right)=-435456 e_{14} \text {. }
$$

(All calculations were made using the computer algebra system Mathematica.)

\section{REFERENCES}

[1] G.M. Bergman, 'Wild automorphisms of free P.I. algebras and some new identities', (preprint) (1981).

[2] V. Drensky and T. Rashkova, 'Weak polynomial identities for the matrix algebra', Comm. Algebra 21 (1993), 3779-3795.

[3] E. Formanek, 'Central polynomials for matrix rings', J. Algebra 23 (1972), 129-132.

[4] A. Giambruno and A. Valenti, 'On minimal *-identities of matrices', Linear and Multilinear Algebra 39 (1995), 309-323.

[5] Ts. Rashkova and V. Drensky, 'Identities of representations of Lie algebras and *-polynomial identities', Rend. Circ. Mat. Palermo 48 (1999), 152-163.

[6] L.H. Rowen, Polynomial identities in ring theory (Academic Press, New York, 1980).

Centre of Applied Mathematics and Informatics

University of Rousse

7017 Rousse

Bulgaria

e-mail: tcetcka@ami.ru.acad.bg 